\title{
Antibiotic Resistance of Isolated Gram Negative Bacteria From Urinary Tract Infections (UTIs) in Isfahan
}

\author{
Mahsa Mirzarazi ${ }^{1}$, Seyedeh Elham Rezatofighi ${ }^{1,}$, Mahdi Pourmahdi ${ }^{2}$, Mohamad Reza \\ Mohajeri $^{3}$ \\ ${ }^{1}$ Department of Biology, Faculty of Science, Shahid Chamran University of Ahvaz, Ahvaz, IR Iran \\ ${ }_{3}^{2}$ Faculty of Veterinary Medicine, Shahid Chamran University of Ahvaz, Ahvaz, IR Iran \\ ${ }^{3}$ Clinical and Pathological Laboratory of Dr. Mohajeri, Isfahan, IR Iran \\ ${ }^{*}$ Corresponding author: Seyedeh Elham Rezatofighi, Department of Biology, Faculty of Science, Shahid Chamran University of Ahvaz, Ahvaz, IR Iran. Tel/Fax: +98-6113331045, E-mail: \\ e.tofighi@yahoo.com.
}

Received: June 24, 2012; Revised: August 25, 2012; Accepted: October 2, 2012

\begin{abstract}
Background: Urinary tract infection (UTI) is a common bacterial disease which may cause chronic renal failure and hypertension. Many reports suggest that the rate of antibiotic resistance to infectious organisms is increasing.

Objectives: This study aimed to detect and also compare the frequency and drug resistance pattern of Gram negative bacteria isolated from patients with community-acquired UTIs in Isfahan.

Patients and Methods: In this cross-sectional descriptive study, 702 samples from 476 females and 226 males referred to medical centers in Isfahan city from June to September 2011 were collected, we investigated the urine cultures and antibiotic sensitivity of the isolated organisms were measured.

Results: Urinary infectious was detected in 203 persons. The most prevalence isolated bacteria were Escherichia coli 138 (68\%), followed by Klebsiella spp. (13\%). Antibiotic resistance pattern of Gram negative bacteria isolated was investigated. Among E. coli isolates the most antibiotic sensitivity and resistance were related to Nitrofurantoin, Cotrimoxazol and Nalidixic acid, Trimetsulpha respectively. Klebsiella spp. isolates were the most antibiotic sensitive to Cotrimoxazol and Cipropheloxacin and the most antibiotic resistant to Trimetsulpha, Cipropheloxacin and Nalidixic respectively.

Conclusions: With regards to the continuous changing in causative microorganisms isolated from patients with UTI and antibiotic sensitivity patterns, it is recommended that bacterial sensitivity patterns in populations are determined in any region annually.
\end{abstract}

Keywords: Urinary Tract Infection; Gram-Negative Bacteria; Antibiotic Resistance

\section{Background}

Urinary tract infection (UTI) represents as one of the most common diseases encountered in medical practices these days and encompasses a broad range of clinical fields that are associated with a common finding of positive urine cultures $(1,2)$. It is the second most common infectious presentation in community. There are an estimated 150 million UTIs per year, worldwide (3, 4). The clinical significance of the infections is due to its high mortality rate, malignant and chronic hypertensions and the chronic renal failures followed by chronic pyelonephritis $(5,6)$. The severity of UTI depends on the bacteria virulence and susceptibility of the host (7). This infection varies in patients with different gender, age, and presence of associated genitourinary abnormalities (8).

UTI is the most common infectious diseases among women $(2,9,10)$. Nearly 1 woman out of 3 , will have at least 1 episode of UTI requiring antimicrobial therapy by the age of 24 years, and almost 50\% of all women will ex- perience at least 1 episode of UTI during their lifetime (11). UTIs are also the most common infections in hospital and extended-care settings, it has been reported that $2 \%-3 \%$ of admitted patients are suffering from UTI $(1,2,12)$.

The etiology of UTIs and the antibiotic susceptibility of urinary pathogens, both in community and hospitals, have been changing over the past years and recently, the antibiotic resistance has become a major global problem $(1,3,7,9)$. There are many reasons for this problem, including antibiotic consumption while feeding the $\mathrm{t}$ animal, inappropriate prescription of antibiotics and poor infection control strategies (1). Due to constant varying of isolated UTI causative microorganisms and antibiotic sensitivity pattern, we recommend to determine bacterial sensitivity in populations every year.

\section{Objectives}

This study is aimed to detect and also compare the frequency and drug resistance pattern of Gram nega-

Implication for health policy/practice/research/medical education:

A variety of antibiotic functions and activities in different regions of this country gives emphasis to a regional surveillance on the activity of antibiotics which should be closely monitored.

Copyright (C) 2013, Ahvaz JundishapurUniversity of Medical Sciences; Published by Kowsar Corp. This is an open-access article distributed under the terms of the Creative Commons Attribution License, which permits unrestricted use, distribution, and reproduction in any medium, provided the original work is properly cited. 
tive bacteria isolated from patients with community acquired UTIs, in Isfahan (Iran).

\section{Patients and Methods}

\subsection{Sample Collection and Analysis}

In this cross-sectional descriptive study, we investigated the urine culture and antibiotic sensitivity of the isolated organisms of the 702 urine samples (476 females and 226 males) of the people refers to medical centers in Isfahan city from June to September 2011. Sampling was performed simply and accidentally. The patients were referred to laboratory or hospital by physicians for detection of urine infection and or abnormality in urine. The study parameters were included the age, sex, bacterial species and bacterial sensitivity or resistance to antibiotics. Isolates were collected without age and sex limitations. Sampling was done from the midstream specimens of urine.

Semi quantitative urine culture using a calibrated loop was performed. Samples were inoculated on a blood agar (Merck, Germany), Mac Conkey agar (Merck, Germany) and Eosin Methylen Blue agar (Merck, Germany) plates. Positive culture was defined as if the bacterial colony count was equal or more than $10^{5} \mathrm{CFU}_{\mathrm{mL}}{ }^{-1}(4,11)$. The species of all isolates were identified $t$ by standard biochemical methods (3). The sampling was blinded and it was not known whether the submitted urinary isolates came from patients with symptomatic upper or lower UTIs or from patients with asymptomatic bacteriuria; however; all submitted isolates had be considered significant by the participating laboratory, identified to species level.

\subsection{Antibiotic Susceptibility Test}

Antimicrobial susceptibility test was performed using disc diffusion method as described by Clinical and Laboratory Standards Institute (CLSI) (13). Briefly, colonies taken from overnight growth on $5 \%$ sheep blood agar (16 $20 \mathrm{~h}$. at $35^{\circ} \mathrm{C}$ ) were re-suspended in Mueller-Hinton broth (Merck, Germany).The turbidity of the suspension is adjusted to an equivalent 0.5 McFarland .This suspension was used to inoculate on Mueller-Hinton agar (Merck, Germany) plates. Cephalexin (CN), Cipropheloxacin (CP), Nalidixic acid (NA), Nitrofurantoin (FM), Trimet-sulpha (SXT), Cefaxim (CFM) and Cotrimoxazol (CTX) (Padtan Teb, Iran) were placed on Mueller Hinton agar .Gram-negative bacilli were incubated at $35^{\circ} \mathrm{C}$ for $16-20 \mathrm{~h}$. and then the inhibition zone was measured and compared with data of Inhibition Zones of Test Cultures by CLSI. Data were interpreted by the percent of susceptible, intermediate, or resistant isolates as defined by CLSI breakpoint interpretative Criteria (13).

\subsection{Statistical Analysis}

Data were analyzed by SPSS software version 16.0, and the Chi-square test, logistic regression and Independent samples t-test performed.

\section{Results}

Of the 702 urine samples processed (476 females and 226 males), 203 samples (28.92\%) were positive for urine culture and had UTI. The mean age was $37.07 \pm 22.2$ years (range from 1 month to 93 years). The prevalence of UTI was observed in females $32.35 \%$ (154 persons) and males $21.68 \%$ (49 persons). This difference was significant statistically $(\mathrm{P} \leq 0.01)$. The infection incidence in women was 1.74 times more than men (95\%, CI: $1.2-2.53$, P $\leq 0.01$ ); so $1.8 \%$ of infection fluctuation was justified by gender (Table 1).

Table 1. Distribution of UTI in Isfahan Patients

\begin{tabular}{lllll}
\hline Age, y & \multicolumn{2}{c}{ Male } & \multicolumn{2}{c}{ Female } \\
\cline { 2 - 5 } & $\begin{array}{l}\text { UTI Posi- } \\
\text { tive, No. } \\
(\%)\end{array}$ & $\begin{array}{l}\text { UTI Nega- } \\
\text { tive, No. } \\
(\%)\end{array}$ & $\begin{array}{l}\text { UTI Posi- } \\
\text { tive, No. } \\
(\%)\end{array}$ & $\begin{array}{l}\text { UTI Nega- } \\
\text { tive, No. } \\
(\%)\end{array}$ \\
\hline o-10 & $4(13.3)$ & $26(86.7)$ & $20(31.7)$ & $43(68.3)$ \\
\hline $\mathbf{1 1 - 3 0}$ & $7(14)$ & $43(86)$ & $44(25.7)$ & $126(74.3)$ \\
\hline $\mathbf{3 1 - 5 0}$ & $14(20.6)$ & $54(79.4)$ & $32(24.8)$ & $97(75)$ \\
\hline $\mathbf{5 1} \leq$ & $24(30.8)$ & $54(69.2)$ & $58(51.3)$ & $55(48.7)$ \\
\hline Sum & $49(21.68)$ & $177(78.32)$ & $154(32.35)$ & $322(67.65)$ \\
\hline
\end{tabular}

Patients were categorized into four different age groups: $\leq 10$ years, $11-30$ years, 31-50 years and $\geq 51$ years (Table 1). Accordingly to Table 1 , UTI is more frequent in older patients , so as much as the patients were elder, the rate of infections was higher. The prevalence of UTI was $28.92 \%$ (95\%, CI: $25.52 \%-32.32 \%$ ). The mean ages of patients with and without infection were 44.07 and 34.28 , respectively. This difference was statistically significant $(\mathrm{P} \leq 0.001)$. The mean age of patients with and without infection was $42.42 \pm 2.11$ and $32.39 \pm 1.04$ in women respectively. This difference is statistically significant $(P \leq 0.001)$. In men, it was $1.67 \pm 37.73$ and $49.27 \pm 3.22$, respectively. This difference was statistically significant $(P \leq 0.001)$. The most microbial agents leading to UTI were Escherichia coli representing 68\% $(\mathrm{n}=138)$ (Table 2$)$, followed by Klebsiella $13 \%(n=27)$, Proteus $4 \%(n=8)$, Pseudomonas $2 \%(n=$ 4) and Gram positive $13 \%(n=26)$ bacteria (Figure 1$)$. The antimicrobial strength of seven antibiotic agents against the most three frequent UTI pathogens was investigated (Table 3). Among the antibiotics, FM had the widest coverage against $E$. coli isolates ( $80 \%$ ) followed by CTX (65\%), CP (57\%), SXT (56\%), CFM (44\%), NA (32\%) and CN (21\%), respectively. The highest resistance rates against NA (63\%) and 
SXT (58\%) were observed among these isolates.

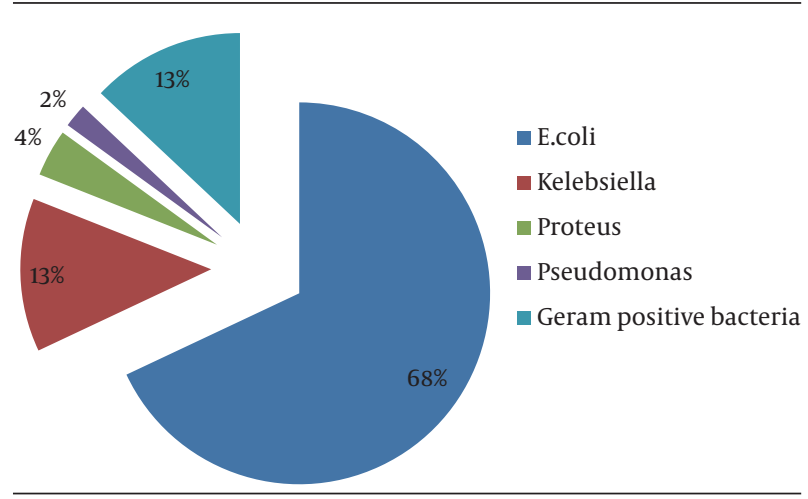

Figure 1. Distribution of the Different Bacteria Isolated From the Urine Samples

For Klebsiella isolates, the antibiotic susceptibility prev- alence is as follow: CTX (74\%), CP (70\%), CFM (63\%), NA (52\%) SXT (48\%) CN(30\%) and Fm (19\%). These isolates had the highest resistance potency against SXT (48\%) and CN (33\%). Among Proteus isolates, CFM , CP and NA were the most active antimicrobial agents, with $89 \%$, 89\% and 78\% effectiveness, respectively; also STX (22\% ) and CTX (22\%) shown the less antimicrobial activity against Proteus isolates.

Table 2. Distribution of E. coli Strains Isolated From UTI in Different Age and Sex Groups

\begin{tabular}{lll}
\hline \multirow{2}{*}{ Age, y } & \multicolumn{2}{c}{ E. coli $(\mathbf{1 3 8}) \mathbf{6 8 \%}$} \\
\cline { 2 - 3 } & Male, No. (\%) & Female, No. (\%) \\
\hline $\mathbf{0 - 1 0}$ & $3(16.7)$ & $15(83.3)$ \\
$\mathbf{1 1 - 3 0}$ & $3(11.2)$ & $24(88.8)$ \\
$\mathbf{3 1 - 5 0}$ & $10(32.3)$ & $21(67.7)$ \\
$\mathbf{5 1} \leq$ & $18(29.04)$ & $44(70.96)$ \\
\hline
\end{tabular}

Table 3. Antibiotic Resistance in Gram Negative Bacteria

\begin{tabular}{llllllllll}
\hline \multirow{2}{*}{ Antibiotic } & \multicolumn{1}{c}{ Bacteria, No. (\%) } \\
\cline { 2 - 10 } & \multicolumn{1}{c}{ Proteus, 9(4) } & \multicolumn{3}{c}{ Klebsiella, 27 (13) } & \multicolumn{3}{c}{ E. coli, 138 (68) } \\
\cline { 2 - 10 } & R & I & S & R & I & S & R & I & S \\
\hline CTXa & $2(22)$ & $1(11)$ & $6(67)$ & $5(19)$ & $2(7)$ & $20(74)$ & $40(29)$ & $8(6)$ & $90(65)$ \\
SXT & $2(22)$ & $1(11)$ & $6(67)$ & $13(48)$ & $1(4)$ & $13(48)$ & $80(58)$ & $2(1)$ & $41(56)$ \\
NA & $2(22)$ & $0(0)$ & $7(78)$ & $8(30)$ & $5(18)$ & $14(52)$ & $86(63)$ & $7(5)$ & $44(32)$ \\
CN & $1(11)$ & $8(89)$ & $0(0)$ & $9(33)$ & $10(37)$ & $8(30)$ & $47(34)$ & $61(45)$ & $29(21)$ \\
CP & $1(11)$ & $0(0)$ & $8(89)$ & $3(11)$ & $5(19)$ & $19(70)$ & $54(39)$ & $5(4)$ & $79(57)$ \\
FM & $2(22)$ & $6(67)$ & $1(11)$ & $6(22)$ & $16(59)$ & $5(19)$ & $8(6)$ & $20(14)$ & $110(80)$ \\
CFM & $1(11)$ & $0(0)$ & $8(89)$ & $8(30)$ & $2(7)$ & $17(63)$ & $47(34)$ & $30(22)$ & $61(44)$ \\
\hline
\end{tabular}

In Table 4 and Figure 2 the rate and percent of resistance E. coli isolates to antibiotics was shown. According to these, more than half of isolates are resistance to 2 or more antibiotics used in this research.

Table 4. The Rate of Multi Drug Resistance (MDR) of E. coli Isolates Collected From UTI

\begin{tabular}{ll}
\hline MDR of E. coli Isolates & No. \\
\hline Total Sensitive & 33 \\
\hline Resistance to 1 antibiotic & 13 \\
\hline Resistance to 2 antibiotics & 31 \\
\hline Resistance to 3 antibiotics & 15 \\
\hline Resistance to 4 antibiotics & 8 \\
\hline Resistance to 5 antibiotics & 14 \\
\hline Resistance to 6 antibiotics & 20 \\
\hline Total Resistance & 4 \\
\hline
\end{tabular}

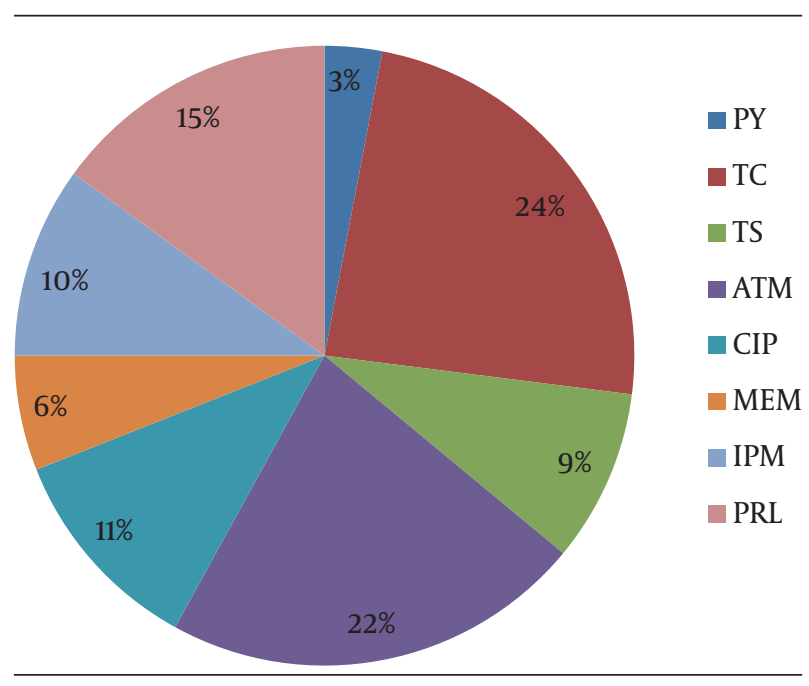

Figure 2. The Rate of Multi Drug Resistance (MDR) of E. coli Isolates Obtained From UTI 


\section{Discussion}

It is important that clinicians are aware of the regional antibiotic resistance rates before initiating experiential antimicrobial therapy for UTI treatment, as it is well-described that urinary infection with a resistant pathogen is more likely to lead to bacteriological/clinical failures (6).

In this study the prevalence of antibiotic resistance of bacterial isolates was investigated. This study was done in Isfahan province, Iran. In this survey UTI in women showed a higher prevalence. In the other studies, like extensively reported results $(1,11)$, this higher prevalence of UTI is due to the anatomic and physical factors in women (11). The highest rate of infection was seen in age group more than 50 years old ( $29 \%$ male and $71 \%$ female).

In some studies, UTI was more detected in babies and adults $(5,14-17)$ but in our study it was different. These results could be due to the increased level of hygiene and public culture and knowledge. Just as shown in Table 2, E. coli (68\%) is the most frequent agent causing UTI. This bacterial agent was reported repeatedly as the most causative agent of UTI $(1,3,4,11,18)$. The other Gram negative and Gram positive bacteria can also be leaded to UTI. Antibiotic resistance is a major clinical problem in treating infections caused by these microorganisms (3). The resistance to the antimicrobials has increased over the years. Resistance rates vary from country to country (3).

The impudence of age, gender and geographic location has previously been shown the amount of antibiotic resistance of urinary isolates (6). According to Table 3 the highest antibiotic resistance of $E$. coli isolates was related to NA (63\%) and STX (58\%). The most effective antibiotics against E. coli were FM (80\%), CTX (65\%) and CP (56\%). The best response to antibiotic therapy was related to FM (80\%). The rate of resistance to this antibiotic in our study was reported $6 \%$ and in North American and Canada was reported $1.1 \%$ and $1.9 \%$, respectively $(4,6)$. In a study in Tehran, Iran, the sensitivity to this antibiotic was reported $86 \%$ and introduced as a selected antibiotic for UTI treatment (5); however in another study in Hamedan, Iran, E. coli isolates were resistance (42.1\%) to this antibiotic (19).

Despite extensive use of FM for many years, this antibiotic still possesses an excellent activity against urinary isolates of $E$. coli $(6,7,11,16,17,19)$; however, compared with fluoroquinolones, the FM is associated with lower treated rates and more side effects in the treatment of acute cystitis $(11,18)$; also, this antibiotic was poorly tolerated by children and old patients and cannot be used for pyelonephritis (5). In our study, after FM, the E. coli isolates were showing the least resistance to CTX. The rate of resistance to this antibiotic was in Europe (14.1\%), USA (18.6\%), Iran (29\%), Spain (33\%), Senegal (55\%), Taiwan (56\%) and India (75\%) (3). In another study in Iran, E. coli isolates were resistance to this antibiotic (19). Resistance, particularly to traditional first line agents such as quinolones and SXT, has increased substantially across Iran. NA is an antibiotic from the first generation of queinolones but nowadays resistance to this antibiotic was reported repeatedly $(7,19)$.

Resistance was reported in Iran and also in our survey (19). CP is an antibiotic from the second generation of flouroqueinolones. According to this subject the functional mechanism of flouroqueinolones is better than FM, so the consumption of these antibiotics for UTI therapy is recommended. Resistance to this antibiotic is also increasing $(6,7,11,19)$. In studies in Tehran and Hamedan respectively $95 \%$ and $93.4 \%$ of bacterial isolates were sensitive to CP but in our study only $57 \%$ of $E$. coli isolates were sensitive to this antibiotic. In some studies $\mathrm{CP}$ was the selected antibiotic for treatment of all of UTI pathogens (11). Klebsiella isolates were shown the least resistance antibiotic (11\%) to CP but in the other regions of Iran such as Hamedan, it is this amount is higher (23\%) (19). In Tehran, Iran Klebsilla spp. showed the highest sensitivity to CP (95.1\%) (16). The sensitivity of Proteus spp. to all antibiotics except CN was seen. In our survey more than $45 \%$ of E. coli isolates were resistance to three or more antibiotics. These results can be showed the unsuitable usage of antibiotics and prevalence of multi drug resistance (MDR) bacteria in Iran. MDR isolates may complicate the therapeutic management of patients with infection by increasing the morbidity and treatment costs by limiting the therapeutic options (4). The difference impacts of these antibiotics, not only in different countries but also in different regions of a country emphasizes that a public and ongoing regional surveillance on the activity of antibiotics and alternative therapies should be closely monitored to assist physicians to the facilate a safe and effective therapy for urinary tract infections.

\section{Acknowledgements}

None declared.

\section{Authors' Contribution}

None declared.

\section{Financial Disclosure}

None declared.

\section{Funding/Support}

The study was supported by Shahid Chamran University of Ahvaz, grant number 89/3/02/44305.

\section{References}

1. Castro-Orozco R, Barreto-Maya AC, Guzman-Alvarez H, OrtegaQuiroz RJ, Benitez-Pena L. [Antimicrobial resistance pattern for gram-negative uropathogens isolated from hospitalised patients and outpatients in Cartagena, 2005-2008]. Rev Salud Publica (Bogota). 2010;12(6):1010-9.

2. Chomarat M. Resistance of bacteria in urinary tract infections. Int J Antimicrob Agents. 2000;16(4):483-7.

3. Akram M, Shahid M, Khan AU. Etiology and antibiotic resistance 
patterns of community-acquired urinary tract infections in J N M C Hospital Aligarh, India. Ann Clin Microbiol Antimicrob. 2007;6:4.

4. Karlowsky JA, Lagace-Wiens PR, Simner PJ, DeCorby MR, Adam HJ, Walkty A, et al. Antimicrobial resistance in urinary tract pathogens in Canada from 2007 to 2009: CANWARD surveillance study. Antimicrob Agents Chemother. 2011;55(7):3169-75.

5. Sharifian M, Karimi A, Tabatabaei SR, Anvaripour N. Microbial sensitivity pattern in urinary tract infections in children: a single center experience of 1,177 urine cultures. Jpn J Infect Dis. 2006;59(6):380-2.

6. Zhanel GG, Hisanaga TL, Laing NM, DeCorby MR, Nichol KA, Weshnoweski B, et al. Antibiotic resistance in Escherichia coli outpatient urinary isolates: final results from the North American Urinary Tract Infection Collaborative Alliance (NAUTICA). Int JAntimicrob Agents. 2006;27(6):468-75.

7. Jadhav S, Hussain A, Devi S, Kumar A, Parveen S, Gandham N, et al. Virulence characteristics and genetic affinities of multiple drug resistant uropathogenic Escherichia coli from a semi urban locality in India. PLoS One. 2011;6(3).

8. Nicolle LE. Epidemiology of urinary tract infections. Clin Microbiol Newsl. 2002;24(18):135-140.

9. Hooton TM, Besser R, Foxman B, Fritsche TR, Nicolle LE. Acute uncomplicated cystitis in an era of increasing antibiotic resistance: a proposed approach to empirical therapy. Clin Infect Dis. 2004;39(1):75-80.

10. Piatti G, Mannini A, Balistreri M, Schito AM. Virulence factors in urinary Escherichia coli strains: phylogenetic background and quinolone and fluoroquinolone resistance. J Clin Microbiol. 2008;46(2):480-7.

11. Karlowsky JA, Jones ME, Thornsberry C, Critchley I, Kelly LJ, Sahm DF. Prevalence of antimicrobial resistance among urinary tract pathogens isolated from female outpatients across the US in
1999. Int J Antimicrob Agents. 2001;18(2):121-7.

12. Mazzulli T. Resistance trends in urinary tract pathogens and impact on management. JUrol. 2002;168(4 Pt 2):1720-2.

13. Wikler MA. Performance standards for antimicrobial susceptibility testing: Sixteenth informational supplement:: Clinical and Laboratory Standards Institute; 2006.

14. Hansson S, Bollgren I, Esbjorner E, Jakobsson B, Marild S. Urinary tract infections in children below two years of age: a quality assurance project in Sweden. The Swedish Pediatric Nephrology Association. Acta Paediatr. 1999;88(3):270-4.

15. Hansson S, Martinell J, Stokland E, Jodal U. The natural history of bacteriuria in childhood. Infect Dis Clin North Am. 1997;11(3):499512.

16. Sharifian M, Karimi A, Tabatabaei SR, Anvaripour N. Microbial sensitivity pattern in urinary tract infections in children: a single center experience of 1,177 urine cultures. Jpn J Infect Dis. 2006;59(6):380-2.

17. Vaezzadeh F, Sharifi-Yazdi MK. Laboratory evaluation of urine culture and drug resistance in children clinically suspected of urinary tract infection (UTI). Iran J Public Health. 2001;30:123-124.

18. Caracciolo A, Bettinelli A, Bonato C, Isimbaldi C, Tagliabue A Longoni L, et al. Antimicrobial resistance among Escherichia coli that cause childhood community-acquired urinary tract infections in Northern Italy. Ital J Pediatr. 2011;37:3.

19. Wagenlehner FM, Weidner W, Naber KG. Emergence of antibiotic resistance amongst hospital-acquired urinary tract infections and pharmacokinetic/pharmacodynamic considerations.J Hosp Infect. 2005;60(3):191-200.

20. Mashouf RY, Babalhavaeji H, Yousef J. Urinary tract infections: bacteriology and antibiotic resistance patterns. Indian Pediatr. 2009;46(7):617-20. 